\title{
Pellegrino Artusi o il Manzoni \\ DELLA CUCINA ITALIANA
}

\author{
Pellegrino Artusi or the Manzoni of the \\ Italian cuisine
}

\author{
Fabiano dalla Bona*
}

\begin{abstract}
RESUMO
La scienza in cucina e l'arte di mangiar bene (A ciência na cozinha e a arte de comer bem), um verdadeiro breviário das massas italianas, a bíblia doméstica de cozinheiros e governantes, um best-seller gastronômico, talvez o maior sucesso editorial italiano de todos os tempos, como disse Piero Camporesi: no transcorrer de quase um século, oito editores publicaram 1.286.000 cópias. Este empreendimento não lhe teria sido possível "se não tivesse sido assistido pela graça da linguagem, se não tivesse adotado aquele italiano especial que lhe permitiu conquistar os fornos do norte ao sul", afirmou Giorgio Manganelli. A língua de um "escritor extraordinário", disse Emilio Tadini. Um escritor que deu vida às Mil e uma noites culinárias, ou, se preferimos, um autêntico Decameron gastronômico. Língua, literatura e identidade são discutidas aqui à luz dos escritos de Piero Camporesi, Alberto Capatti e Giovanna Frosini.
\end{abstract}

Palavras-chave: Pellegrino Artusi; língua italiana; gastronomia.

\footnotetext{
ABSTRACT

La scienza in cucina e l'arte di mangiar bene (The Science in the Kitchen and the Art of Eating Well), a true breviary of Italian

${ }^{*}$ UFRJ.
} 
pasta, the domestic bible of cooks and rulers, a gastronomic bestseller, perhaps the most successful Italian publication of all time, as said by Piero Camporesi: over the course of nearly a century, eight editors published $1,286,000$ copies. This project would not have been possible "if it had not been assisted by the grace of language, if it had not adopted that special Italian that allowed him to conquer the ovens from north to south," said Giorgio Manganelli. The language of an "extraordinary writer," said Emilio Tadini. A writer who gave life to the culinary Thousand and One Nights, or, if we prefer, an authentic gastronomic Decameron. Language, literature and identity are discussed here in the light of the writings of Piero Camporesi, Alberto Capatti and Giovanna Frosini.

Keywords: Pellegrino Artusi; Italian language; gastronomy.

Diffidate dei libri di cucina che trattano di quest'arte: sono la maggior parte fallaci o incomprensibili, specialmente quelli italiani; meno peggio i francesi: al più al più, tanto dagli uni che dagli altri, potrete attingere qualche nozione utile quando l'arte la conoscete.

Pellegrino Artusi ${ }^{1}$

Mi permetto di iniziare con le parole dello storico Massimo Montanari: "L'Italia delle cento città e dei mille campanili è anche l'Italia delle cento cucine e delle mille ricette." (MONTANARI; CAPATTI, 1999, VII).

Nell'Italia appena fatta del 1891, c'erano da fare gli italiani, come aveva detto anni prima il piemontese Massimo D'Azeglio (1798-1866). Nella sua celebre formula, D'Azeglio, non solo uomo politico di primo piano ma anche scrittore di successo e non mediocre pittore, concretizzò lucidamente un autentico problema della nuova Italia, anzi concretizzò "il problema". Infatti, perché il neonato Stato potesse affondare solide radici e potesse sopravvivere e rafforzarsi, era necessario che tutti coloro che lo abitavano, o almeno la maggior parte, riconoscessero di condividere qualcosa, sentissero di appartenere ad una stessa storia, ad una stessa lingua, facessero riferimento ad un patrimonio comune di valori, di miti, di sogni, di affetti, di predilezioni, e ovviamente, di sapori e di saperi.

${ }^{1}$ Tutte le citazioni del presente paper si rifanno alla seguente edizione: ARTUSI, Pellegrino. La scienza in cucina e l'arte di mangiare bene. Torino: Einaudi, 2001. 
A Firenze, dove viveva il nostro autore, ancora vivo era il ricordo della Capitale d'Italia. Insomma era vivo un forte spirito nazionale, una voglia d'Italia e di Patria. Si era formata quella che chiamano la "borghesia unitaria" che partecipava alla costruzione dell'identità nazionale senza ripudiare il regionalismo, ma cercando di mediarlo nei nuovi confini. Il nostro Artusi, nel suo piccolo, si pose prima di tutto un problema di lingua.

Per comprendere il ruolo che l'Artusi ebbe nella formazione degli Italiani, bisogna partire dal fatto che il suo libro fu venduto in tutta la Penisola, che milioni di donne se ne servirono, che passò di casa in casa, di generazione in generazione. Quindi ebbe um impatto diffuso e duraturo, ben al di là di quanto è testimoniato dal numero di edizioni e di copie, che pure è altissimo. Si parla oggi di oltre tre milioni di copie vendute! Un vero best seller. Il critico letterario e antropologo Piero Camporesi, nel saggio introduttivo all'edizione Einaudi del 1970, così scrive sull'opera in analisi:

Un manuale che non è soltanto un libro di cucina come tanti altri: acanto a Cuore (e a Pinocchio) è uno dei massimi prodotti della società italiana del secondo Ottocento, una di quelle bibbie popolari che il moderatismo sociale italiano ha espresso per la costruzione di un cittadino fabbricato secondo i postulati dell'etica borghese, corroborato da uno slancio e da una serietà che la letteratura sociale non riuscirà più a ritrovare. (CAMPORESI, 2001, p. XXIII).

\section{E aggiunge:}

Limportanza dell'Artusi è notevolissima e bisogna riconoscere che La scienza in cucina ha fatto per l'unificazione nazionale più di quanto non siano riusciti a fare i Promessi Sposi. I gustemi artusiani, infatti, sono riusciti a creare un codice di identificazione nazionale lá dove fallirono gli stilemi e i fonemi manzoniani. Ciò, si capisce, anche perché non tutti leggono mentre tutti, al contrario, mangiano; nel caso poi del sucesso artusiano la cosa è ancor più educativa perché si mangia ciò che prima si è letto, rovesciando la priorità dell' antico "nil est in intellectu quod non fuerit prius in sensibus" e dimostrando, se ocorresse, che la cucinaria è la più antica forma di cultura popolare, per eccellenza orale, anche se di una oralità incoativa. (CAMPORESI, 2001, p. XXII).

Secondo Lévi-Strauss, i gustemi sono le unità minime colme di significato in un particolare contesto umano. L'universo del gusto, e così, dei gustemi, va riferito, in essenza, al tema dell'identità culinaria delle società, dietro al quale troviamo la dimensione del suo significato profondo 
fra il colletivo e l'individuale. Afferma Lévi-Strauss che "come la lingua, mi sembra che la cucina di una società sia analizzabile in elementi costitutivi che si potrebbero chiamare 'gustemi', i quali sono organizzati secondo talune strutture di opposizione e di correlazione." (LÉVI-STRAUSS, 2009, p. 103).

Proprio per tali strutture, e per la diffusione e la persistenza, l'opera artusiana fu in grado di influenzare la lingua settoriale dell'alimentazione, contribuendo non poco alla formazione di un lessico comune, di un vocabolario condiviso. Infatti, l'eredità di divisioni dell'Italia, aveva fatto sì che esistessero molteplici nomi diversi, a seconda delle regioni, per gli stessi attrezzi da cucina, per gli stessi tagli di carne, per le stesse modalità di preparazione, per gli stessi piatti. Pellegrino Artusi era consapevole che considerarsi un unico popolo significava anche far uso di una lingua comune a tavola. A proposito della ricetta del caciucco, egli rilevava come questo stesso piatto fosse chiamato in modo diverso nelle varie regioni della Penisola:

La confusione di questi e simili termini fra provincia e provincia in Italia, è tale che poco manca a formare una seconda Babele. Dopo l'unità della patria mi sembrava logica conseguenza il pensare all'unità della lingua parlata, che pochi curano e molti osteggiano, forse per falso amor proprio e forse anche per la lunga ed inveterata consuetudine ai proprii dialetti. (ARTUSI, 2001, p. 399).

Grazie al suo libro, questa situazione di frammentazione fu superata, anche se non del tutto, tanto che i numerosissimi libri di ricette che ogni anno vengono pubblicati sanno di poter contare su un comune lessico che qualsiasi lettore possiede, e che gli stessi libri sono, in qualche modo, dei suoi debitori. Ed è interessante notare come, con il passare del tempo, Artusi diventa il titolo del libro laddove il vero titolo viene dimenticato. Addirittura viene dizionarizzato:

Artusi: per antonomasia libro di cucina. Che gloria! Il libro che diventa nome! A quanti letterati toccò tale sorte? Era l'Artusi di Forlimpopoli (1821-91 [sic]), banchiere, cuoco, bizzarro, caro signore, e molto benefico, come dimostrò nel suo testamento; e il suo trattato è scritto in buon italiano. E non era letterato né professore. (PANZINI, 1905, p. 23).

In secondo luogo, questo libro, che pur si fonda sulle conoscenze sperimentate direttamente dall'autore e, quindi, sul patrimonio gastronomico soprattutto dell'Italia centrale, si presenta anche come un primo tentativo di proporre una cucina nazionale, una cucina degli italiani per gli italiani. 
In tal modo, Artusi avviò una specie di superamento delle barriere regionali e insegnò alle donne del Sud a fare le ricette del Centro e del Nord e viceversa. È la proposta di un interscambio di consuetudini alimentari, di conoscenze, una familiarizzazione, un'appropriazione dell'altro, un primo e fondamentale passo verso la nascita della "cucina italiana".

E ancora: con la sua enorme diffusione Artusi contribuì a far nascere e a diffondere una cultura comune nel paese e l'idea che il cibo non serve soltanto a recuperare le energie, ma è arte, scienza, piacere e cultura.

Come è saputo, soprattutto in cucina, la lingua che dominava in quell'epoca era il francese, anche nelle cucine italiane, e soprattutto nei libri di cucina, abbondavano i termini francesi, italianizzati, mal tradotti o ibridati: insomma un grande miscuglio linguistico. Un patriota della pentola quindi, doveva porsi il problema. Non per combattere contro i grandi maestri francesi, ma per offrire alla borghesia italiana un comune terreno di comprensione. Gli fu complice e sostenitore lo scrittore Olindo Guerrini, con cui il nostro scambiò gustose lettere che ironizzavano sulla moda delle sgangherate traduzioni dal francese.

Secondo lo storico Alberto Capatti,

Artusi combatte i titoli misteriosi, le denominazioni sussiegose, e prende a bersaglio, uno per tutti, il francese. Trattandolo alla stessa guisa dei dialetti, con estrema naturalezza traduce i beignets in ciambelline, e la fondue in cacimperio. Per pura civetteria, lascia in francese la "Salsa alla maître d'hôtel". [...] La lingua francese è petulante, boriosa, superflua. Questa conclusione è ribadita ripetutamente, con mille sottigliezze. (CAPATTI, 2009, p. 14).

Nell'operazione linguistica dell'Artusi troviamo anche degli errori di ortografia; forse perché l'italiano, anche se incerto e oriundo di traduzioni, era da lui ritenuto sempre migliore rispetto alla lingua straniera, nel caso il francese. Parliamo di uno schiaffo a quella lingua della cultura gastronomica ottocentesca. Infatti, Artusi usa la parola soufflet, così grafata, e verseggia, proponendo i neologismi sgonfiotto e tramesso nella ricetta Sgonfiotto di farina gialla (682):

Questo piatto

I francesi lo chiaman soufflet

E lo notano come entremet, Io sgonfiotto, se date il permesso,

Che servire potrà di tramesso. (ARTUSI, 2001, p. 605). 
Entremet senza la $s$ e soufflet con una $t$ inesistente. Il primo forse è un errore di ortografia e il secondo pure, visto che scritta così la parola significa schiaffo, sberla. A dirla con Capatti, "un lapsus o una seconda malizia?" Secondo la storica della lingua Giovanna Frosini,

Qui Artusi poteva giovarsi dell'autorità del suo amato Fanfani, che registra, sgonfiotto nel senso di 'morseletto di pasta dolce, che friggendosi si rigonfia, ed è di ottimo gusto; ma questa parola aérea non è sopravvissuta, ed è stata senza scampo sovrastata dal termine francese originario. (FROSINI, 2009, p. 5).

Ma c'era anche il "problema", quello di costruire una lingua italiana in un paese in cui, se si escludeva l'élite intellettuale, la gente parlava ancora in dialetto, anzi, un paese in cui esistevano ed andavano per la maggiore, libri di cucina regionale scritti in dialetto. Non a caso l'Artusi fa precedere il libro vero e proprio da una "Spiegazione di voci che essendo dal volgare toscano non tutti intenderebbero" .

Artusi conosceva il francese, praticava il fiorentino parlato e scritto, conosceva la terminologia di base di molti dialetti e voleva farsi comprendere da tutti gli italiani. "In un clima di nazionalismo patriottico e di marcata sensibilità pedagogica” (MONTANARI; CAPPATI, 2005, p. 239), Artusi non si lanciò in una crociata dissennata contro il francese, ma si assegnò il ruolo di mediatore tra l'italiano e le tradizioni dialettali: non volle semplicemente cancellare i francesismi, quanto piuttosto costruire un italiano della cucina, arricchito da voci toscane e dialettali, servili e professionali, di uomini e donne. E sottolinea Cappati:

La raccolta di ricette di Pellegrino Artusi, conosciuta col titolo di Scienza in cucina e stampata nel 1891, non è solo il frutto degli ozi di un ricco borghese romagnolo e fiorentino, ma un opera di impegno civile : istruire cuoche e cuochi nella lingua italiana, far loro conoscere il patrimonio di molte regioni italiane, dalla Sicilia al Piemonte, stimolare una attenzione patriottica al cibo contro l'imperante francofilia. (CAPATTI, 2010, p. 12).

\footnotetext{
${ }^{3}$ Wilson, Jackie. The Very Best of Jackie Wilson, Ace Records UK, 2004. A canção pode ser ouvida no seguinte link: http://www.youtube.com/watch?v=xJ3-NnNx6Zs

${ }^{4}$ Aragão, Jorge. Roda de Samba com Jorge Aragão. Som Livre, 2006. A canção pode ser ouvida no seguinte link: http://www.youtube.com/watch?v=cxmFZDppfKo
} 
L'uso di una lingua facile ma garbata, familiare ma quasi narrativa, la scelta della borghesia come pubblico di riferimento, la capacità di esplorare i patrimoni gastronomici italiani e renderli accessibili a tutti, garantirono alla Scienza in cucina un ruolo centrale nella formazione del gusto italiano del Novecento.

Artusi, dunque, affondò la sua scrittura nel volgare toscano, soprattutto in quello fiorentino, e tale scelta si rivelò vincente, e la sua divenne una sorta di koinè culinaria; fu l'Artusi a parlare per primo della bistecca alla fiorentina, ad esempio.

Artusi, come dice lo storico Eric Hobsbawn, "inventa la tradizione": ${ }^{2}$

Le lingue nazionali imparate a scuola, per essere scritte - lasciamo da parte la lingua parlata - da qualcosa di più che un'élite piuttosto ristretta, sono per lo più il prodotto di un'epoca dalla durata variabile, ma quasi sempre breve. Come osservava giustamente uno storico francese della lingua fiamminga, il fiammingo che si insegna oggi in Belgio non è quello che parlavano le mamme e le nonne di Fiandra coi loro bambini: in poche parole, è una "madrelingua" in senso metaforico, non letterale. Non dobbiamo lasciarci fuorviare da un paradosso curioso, ma comprensibile: in genere le nazioni moderne, con tutto il loro armamentario, pretendono di essere l'opposto della novità, si dichiarano radicate nell'antichità più remota, stanno al polo opposto delle comunità costruite, cioè umane, sono tanto "naturali" da non richiedere altra definizione che l'autoaffermazione. (HOBSBAWM, 2002, p. 20).

Con la sezione dedicata a "Spiegazione di voci, che essendo del volgare toscano, non tutti intenderebbero" entriamo nel fulcro della questione linguistica. L'autore affronta il paziente lavoro di linguista, e non come un apprendista, anzi da esperto, e si avvale degli strumenti più adatti, come egli stesso dichiara, pure in modo scherzoso, citando la Crusca come unica e vera autorità linguistico-letteraria indiscussa, e al cui dizionario si affida per discernere fra i termini accreditati dalla tradizione e quelli spuri.

Oggi, le trentasei voci ordinate alfabeticamente nel libro, potrebbero suonare perfettamente italiane e non più vernacolari, ad eccezione di pocchissime. Ha avuto notevole fortuna diffondendosi per tutta la penisola, la parola bietola, che secondo De Mauro e Mancini (DE MAURO; MANCINI,

\footnotetext{
${ }^{2}$ Per "tradizione inventata" si intende un insieme di pratiche, in genere regolate da norme apertamente o tacitamente accettate, e dotate di una natura rituale o simbolica, che si propongono di inculcare determinati valori e norme di comportamento ripetitive nelle quali è automaticamente implicita la continuità col passato. HOBSBAWN, Eric J. L'invenzione della tradizione. Torino: Einaudi, 2002, p. 16.
} 
2000 , p. 237) risale al 1310; fagiuoli, ormai persa la dittongazione, già ampiamente affermata all'epoca e risalente al 1205 (DE MAURO; MANCINI, 2000 , p. 731). Meno comuni i termini che indicano le interiora, le frattaglie ormai difusissimo contro cipolla, "che parlando di polli vale ventriglio", pasto come "polmone dei quadrupedi", pietra chiosato con "rognone, arnione" che non hanno avuto la stessa forza nell'affermarsi, vinti lessicalmente dagli antagonisti ventriglio, polmone, rognone che si sono imposti alle varianti regionali. Curiosa la denominazione frattagliaio con il tipico sufisso italiano -aio, contrapposto ai forestierismi -iere e -ista, sottolineando il livello sociale di colui che svolgeva l'attività. Questo mestiere, ormai quasi scomparso, sopravvive ancora nel napoletano e nel ligure come trippaio o trippaiolo, secondo il dizionario di Gabriele Aldo (ALDO, 2011, p. 1018). Ancora per quanto riguarda la carne, troviamo carnesecca per "pancetta di maiale salata", ormai diventata soltanto pancetta. Troviamo ancora costoletta che diversamente dall'uso odierno che indica la costola accompagnata solo dalla carne che la riveste, che l'Artusi vuole come braciola colla costola, e appunto braciola è prevalso; cotoletta che sarebbe un "francesismo", così come avvertito dall'autore, ma da allora univoco per indicare la preparazione in questione, risalente al 1597 (DE MAURO; MANCINI, 2000, p. 493); filetto per indicare il taglio tanto di carne come di pesce, così come lo capiamo ancora oggi. Già il lardo come strutto di maiale e "che a Napoli si chiama nzogna", lardone ormai caduto in disuso, contrapposto al termine strutto ancora in uso e da lui scartato, e perfino sugna, all'epoca un regionalismo che ormai è perfettamente comprensibile, e per finire, lardo vergine mai usato oggi. Scaloppe o scaloppine per fetta sottile di carne magra, si afferma ed è ancora in uso, derivato dal francese, di origine incerta e risalente al 1890, dunque, al libro di Artusi. (DE MAURO; MANCINI, 2000, p. 1860)

Nel campo delle preparazioni di base abbiamo bianchire che il proprio Artusi rimanda a imbiancare, raramente usate all'epoca, un volta superata dalla voce scottare, ma che è tornata all'uso di recente e viene molto utilizzate oggi nel senso di "schiarire carne o verdura, scottandola in acqua bollente e raffredandola" (ZINGARELLI, 1989, p. 470), panare oggi impanare.

Tra gli arnesi di cucina troviamo il lardatoio che conserva ancora oggi lo stesso significato e lo stesso nome ma poco usato, dal momento che col termine steccare si designa precisamente l'azione e si sottintende lo strumento col quale la si compie; la lunetta o mezzaluna che conservano ancora nome e significato, con una certa prevalenza del secondo sul primo, così come matterello, risalente al 1755 (DE MAURO; MANCINI, 2000, p. 1192) talvolta chiamato anche mattarello con l'abbassamento della vocale in sillaba 
pretonica; il mestolo che si è imposto sui regionalismi come ramina, cassa o cucchiaia; la spianatoia originalmente una voce toscano-romagnola ormai diffusa su tutto il territorio, e non confondibile con il tagliere che il proprio Artusi vuole di piccola dimensione e lo staccio oggi setaccio.

Oggi chiamiamo toscanismo quel suo scrivere "garbare" per piacere, "stuccare" per venire a noia, "diacciare" per ghiacciare, "stiacciata" per schiacciata, "infreddatture" per raffreddori, "lardellare" per avvolgere con il lardo, "alidire" per far sfumare, "gli odori" per le erbe aromatiche. Termini desunti dal linguaggio gastronomico fiorentino e toscano, "come la parola arista per il lombo del maiale, di origine greca, ma appartiene alla tradizione toscana, ed è molto più antica di quanto pensi Artusi, che la lega al Concilio fiorentino del 1439, invece ha una continuità semantica lunghissima, fin dai tempi di Dante e non ha mai cambiato significato" (FROSINI, 2009, p. 5) prosegue la studiosa.

Egli, in realtà, si limitò a raccogliere, tramite fonti orali o scritte, quanto di meglio trovò nelle varie tradizioni regionali dell'Italia unita, e in particolare dell'Emilia-Romagna e della Toscana, le realtà che meglio conosceva. Intervenne attivamente, modificò, adattò, corresse: tutto per rendere accettabili e comprensibili le sue ricette al pubblico di tutta Italia. Concorse a realizzare sul piano gastronomico quell'unità culturale (e anzitutto linguistica) che sembrava irraggiungibile. Si rivolse espressamente alle classi agiate, ma la novità del suo stile narrativo e non normativo, la capacità di instaurare col lettore una sorta di dialogo confidenziale, ne decretarono il grande successo, in un mondo editoriale che cominciava già a pullulare di volumi sulla cucina, il più delle volte, complicati e tediosi.

"Pellegrino Artusi segna la nascita della cucina italiana moderna, che si afferma non solo fra le borghesie cittadine, prime destinatarie della Scienza in cucina, ma, col tempo, anche fra le classi popolari", sostiene Montanari. (MONTANARI, 2010, p. 57)

E aggiunge lapidariamente:

Se ancora oggi, dopo oltre un secolo dalla sua prima edizione, $\mathrm{La}$ scienza in cucina si stampa, si legge, si discute, è perché Artusi seppe guardare lontano, giocando d'anticipo su tendenze che si sarebbero pienamente affermate nella cucina italiana del Novecento, ma che nel 1891 erano ancora in fieri. (MONTANARI, 2010, p. 58). 


\section{RIFERIMENTI BIBLIOGRAFICI}

ARTUSI, P. La scienza in cucina e l'arte di mangiare bene. Torino: Einaudi, 2001.

. La scienza in cucina e l'arte di mangiare bene. Milano: Antonio Vallardi Editore, 2009. . La scienza in cucina e l'arte di mangiare bene. Firenze: Giunti Editori, 2011.

CAMPORESI, P. Introduzione. In: ARTUSI, P. La scienza in cucina e l'arte di mangiare bene. Torino: Einaudi, 2001. p. XV-LXXVIII.

CAPATTI, A. Il boccone immaginario: saggi di storia e letteratura gastronomica. Bra: Slow Food Editore, 2010.

. Lingua e letteratura nell'Artusi: il libro che ha fondato la cucina italiana. Atti del convegno Lingua e letteratura nell'Artusi, Firenze, 28/03/2009. Disponível em: <www.pellegrinoartusi.it>. Acesso em: 20/02/2011.

DE MAURO, T.; MANCINI, M. Dizionario etimologico. Milano: Garzanti, 2000.

FROSINI, G. Lamore per la lingua, la pratica della lingua nella Scienza in cucina. Atti del convegno Lingua e letteratura nell'Artusi. Firenze, 28/03/2009. Disponível em: <www.pellegrinoartusi.it>. Acesso em: 20/02/2011.

GABRIELLI, A. Grande dizionario italiano. Milano: Hoepli, 2011.

HOBSBAWM, E. J. L'invenzione della tradizione. Torino: Einaudi, 2002.

LÉVI-STRAUSS, C. Antropologia strutturale. Milano: Il Saggiatore, 2009.

MONTANARI, M.; CAPATTI, A. La cucina italiana. Roma-Bari: Laterza, 2005.

. L'identità italiana in cucina. Roma-Bari: Laterza, 2010.

PANZINI, A. Dizionario moderno. Milano: Hoepli, 1905.

SABATINI, F.; COLETTI, V. Dizionario della lingua italiana. Milano: Rizzoli, 2006.

ZINGARELLI, N. Vocabolario della lingua italiana. Torino: Loescher, 1989.

Submetido em: 06/11/2012

Aceito em: 13/02/1013 